\title{
In vivo Reflectance Confocal Microscopy: A Useful Tool to Select the Location of a Punch Biopsy in a Large, Clinically Indistinctive Lesion
}

\author{
Malou Peppelman ${ }^{a} \quad E_{\text {sther A.W. Wolberink }}{ }^{a} \quad$ Roland J.J. Koopman $^{b}$ \\ Piet E.J. van Erp ${ }^{a} \quad$ Marie-Jeanne P. Gerritsen ${ }^{a}$ \\ aDepartment of Dermatology, Radboud University Nijmegen Medical Centre, Nijmegen, \\ and ${ }^{\mathrm{b}}$ Department of Dermatology, Bernhoven Hospital, Oss, The Netherlands
}

Key Words

Squamous Cell carcinoma · Diagnosis · Biopsy · Reflectance confocal microscopy

\begin{abstract}
Reflectance confocal microscopy (RCM) is a noninvasive technique for in vivo imaging of the skin that allows evaluation of the total lesion area. This case report about a 66-year-old patient with a clinically indistinctive, previously treated erythematous lesion on the frontal part of the face demonstrates the use of RCM to select the proper biopsy location.
\end{abstract}

\section{Introduction}

Squamous cell carcinoma (SCC) is the second most common nonmelanoma skin cancer after basal cell carcinoma (BCC) [1,2]. Currently, diagnosis of a clinically suspect SCC or BCC is confirmed by histological evaluation of a skin biopsy obtained from the clinically most suspicious part of the lesion. However, a biopsy only represents a small part of the lesion. Especially in large lesions, this might result in a false-negative diagnosis or in missing a more aggressive part of the lesion [3, 4]. Furthermore, clinical evaluation of previously treated and/or large lesions can be difficult, and in such cases, it is hard to determine the most suspect area for a biopsy.

Reflectance confocal microscopy (RCM) is a noninvasive technique for in vivo imaging of the skin that allows evaluation of the total lesion area [5]. It is known that RCM can visualize nonmelanoma skin cancer, melanoma and inflammatory skin diseases [6, 7]. However, to the 
best of our knowledge, it is not described whether RCM might be used to select the location of a punch biopsy in a large and clinically indistinctive lesion.

\section{Case Presentation}

A 66-year-old patient with an erythematous lesion on the frontal part of the face was referred from the Department of Dermatology of the Bernhoven Hospital, Oss, The Netherlands. At this hospital, the lesion was treated with cryotherapy followed by Efudix. Unfortunately, both treatments were ineffective. A punch biopsy after these therapies revealed the presence of a superficial BCC and, therefore, the lesion was treated with photodynamic therapy. However, the lesion persisted and increased in size. The biopsies obtained after photodynamic therapy showed chronic inflammation and no residual tumor tissue. For further management, the patient was referred to the outpatient clinic of the Department of Dermatology, Radboud University Nijmegen Medical Centre, Nijmegen, The Netherlands. A clinical diagnosis of the erythematous lesion with a size of $2.3 \times 2.1 \mathrm{~cm}$ was difficult due to the previously performed biopsies and treatments (fig. 1). This made it challenging to determine the most suspect area for a biopsy. Treatment by surgical excision without prior establishment of the diagnosis was unfavorable due to the size and the location of the lesion. Therefore, we used RCM for a noninvasive evaluation of the complete lesion to select the most suspect area for a biopsy.

The major part of the lesion showed RCM features corresponding to normal skin or actinic keratosis. More importantly, one small part showed prominent aberrant features: a disrupted stratum corneum, keratinocyte atypia, spongiosis, increased capillary blood flow, flattening of the dermal epidermal junction, solar elastosis and a nest of atypical cells (fig. 2). These features were suspect for either SCC or BCC. A 3-mm punch biopsy was taken from this part of the lesion. Histopathological evaluation of the biopsy showed the presence of a SCC with an invasive growth of $0.9 \mathrm{~mm}$. The lesion was excised and evaluation of the excision specimen showed multifocal actinic keratosis and two small foci displaying SCC with an invasive growth of 0.8 and $0.5 \mathrm{~mm}$.

\section{Conclusion}

Although it is known that RCM can be helpful for the diagnosis of nonmelanoma skin cancer, this case demonstrates the practical use of RCM as a tool to select the location for a punch biopsy in a large and clinically indistinctive lesion. In this case, the foci displaying SCC were only a small part of the total lesion area. Without RCM imaging prior to the biopsy, the most aggressive part of the lesion could have been missed. RCM allows evaluation of the total lesion area, which might reduce sample errors and delay in accurate diagnosis and treatment of large or clinically indistinctive lesions.

\section{Disclosure Statement}

The authors have no conflicts of interest to declare. 
Peppelman et al.: In vivo Reflectance Confocal Microscopy: A Useful Tool to Select the Location of a Punch Biopsy in a Large, Clinically Indistinctive Lesion

\section{References}

1 Lomas A, Leonardi-Bee J, Bath-Hextall F: A systematic review of worldwide incidence of nonmelanoma skin cancer. Br J Dermatol 2012;166:1069-1080.

2 Ridky TW: Nonmelanoma skin cancer. J Am Acad Dermatol 2007;57:484-501.

3 Haws A, Rojano R, Tahan SR, Phung T: Accuracy of biopsy sampling for subtyping basal cell carcinoma. J Am Acad Dermatol 2012;66:106-111.

4 Wolberink EA, Pasch MC, Zeiler M, van Erp PE, Gerritsen MJ: High discordance between punch biopsy and excision in establishing basal cell carcinoma subtype: analysis of 500 cases. J Eur Acad Dermatol Venereol 2012, E-pub ahead of print.

5 Calzavara-Pinton P, Longo C, Venturini M, Sala R, Pellacani G: Reflectance confocal microscopy for in vivo skin imaging. Photochem Photobiol 2008;84:1421-1430.

6 Guitera P, Menzies SW, Longo C, Cesinaro AM, Scolyer RA, Pellacani G: In vivo confocal microscopy for diagnosis of melanoma and basal cell carcinoma using a two-step method: analysis of 710 consecutive clinically equivocal cases. J Invest Dermatol 2012;132:2386-2394.

7 Ulrich M, Lange-Asschenfeldt S, González S: In vivo reflectance confocal microscopy for early diagnosis of nonmelanoma skin cancer (article in English, Spanish). Actas Dermosifiliogr 2012;103:784-789.

M.P. and E.A.W.W. contributed equally to this work.

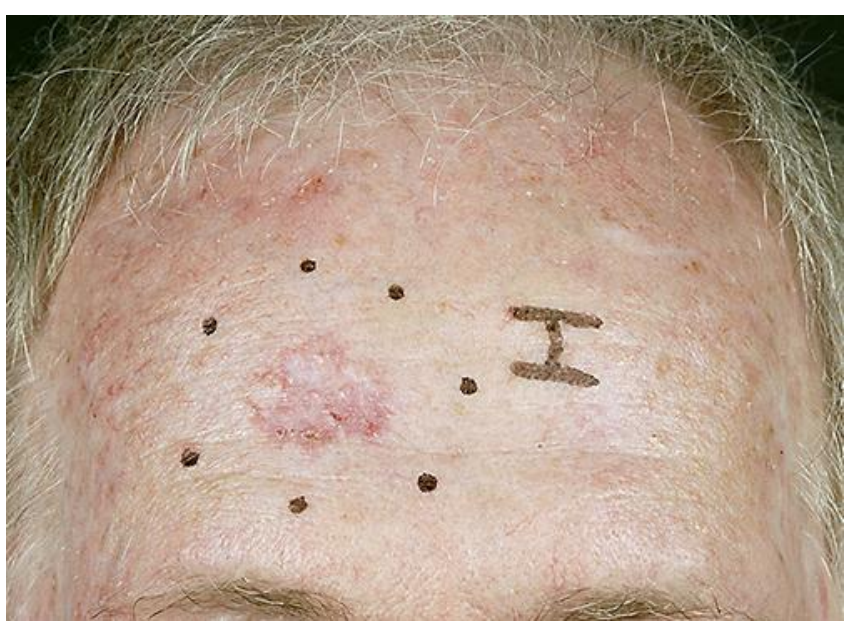

Fig. 1. Clinical picture of an erythematous plaque located on the frontal area of the face. The lesion showed a heterogeneous appearance with erythema, mild induration and an indistinct margin. The lesion had a size of $2.3 \times 2.1 \mathrm{~cm}$ and had been present for over 10 years. 


\section{Case Reports in Dermatology}

\begin{tabular}{l|l}
\hline Case Rep Dermatol 2013;5:129-132 & \\
\hline DOI: 10.1159/000351258 & $\begin{array}{l}\text { @ 2013 S. Karger AG, Basel } \\
\text { www.karger.com/cde }\end{array}$ \\
\hline
\end{tabular}

Peppelman et al.: In vivo Reflectance Confocal Microscopy: A Useful Tool to Select the Location of a Punch Biopsy in a Large, Clinically Indistinctive Lesion
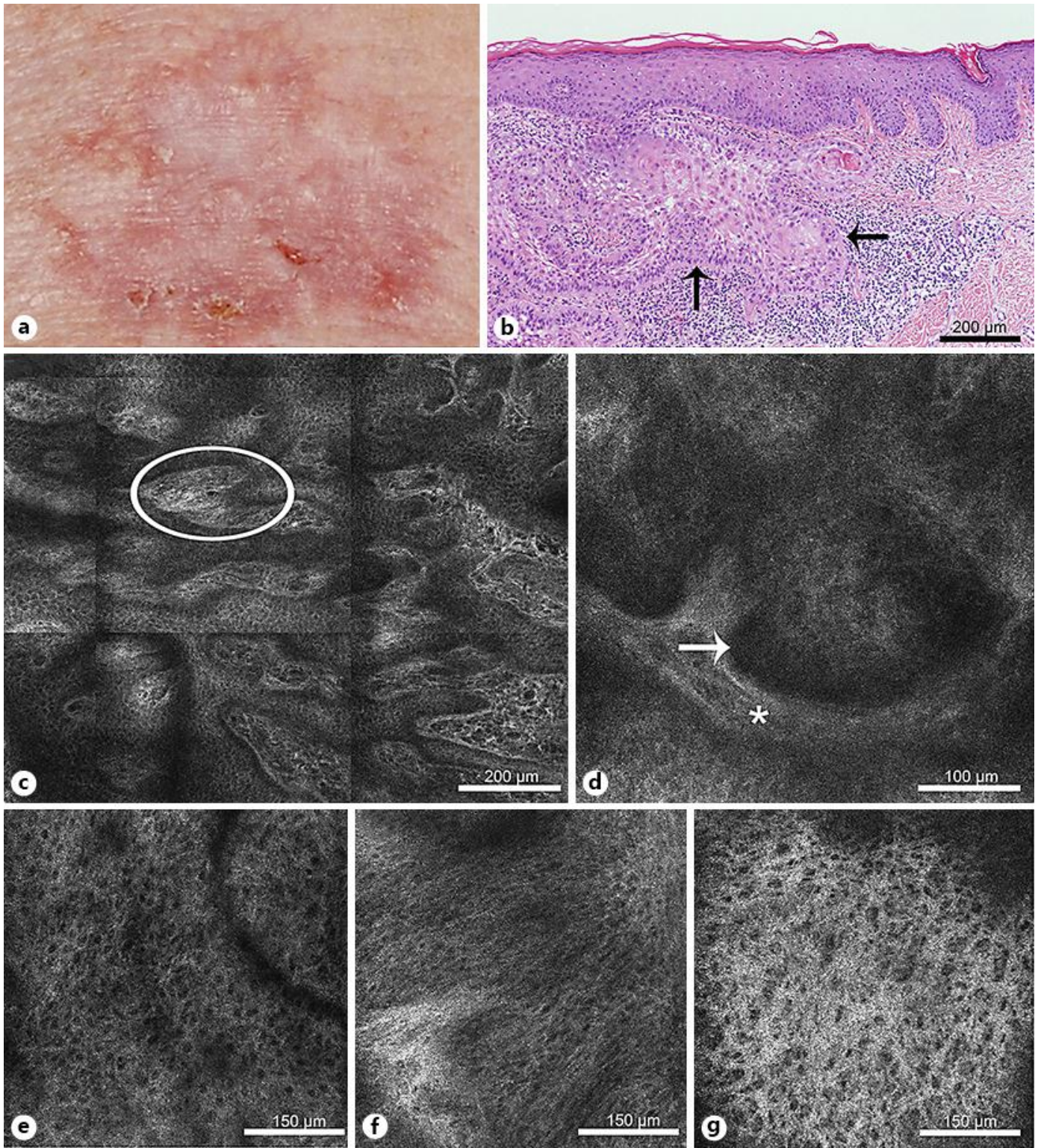

Fig. 2. Clinical and RCM images of the SCC with the corresponding histology. a Clinically, an erythematosquamous plaque with an irregular border was located on the frontal area of the face. Depigmentation was mainly found in the cranial part of the lesion. b Corresponding hematoxylin-eosin stained tissue section displaying an invasive SCC (black arrows). c RCM image showing flattening of the dermal epidermal junction. Islands of dermal cells (encircled area) are interspersed between epidermal areas. $\mathbf{d}$ Tumor nest consisting of atypical epidermal cells (white arrow) surrounded by fibrosis (asterisk). e Spongiosis, visualized as enlargement of the bright intercellular spaces. $f$ Keratinocyte atypia at the stratum spinosum. g Severe solar elastosis was seen in the dermis, visualized as coarse, highly reflective collagen bundles. 